\title{
A P-Lingua Programming Environment for Membrane Computing
}

\author{
Daniel Díaz-Pernil, Ignacio Pérez-Hurtado, \\ Mario J. Pérez-Jiménez, Agustín Riscos-Núñez. \\ Research Group on Natural Computing \\ Dpt. Computer Science and Artificial Intelligence. University of Sevilla \\ Avda. Reina Mercedes s/n. 41012 Sevilla, Spain \\ $\{$ sbdani, perezh, marper, ariscosn $\}$ @us.es
}

\begin{abstract}
A new programming language for membrane computing, PLingua, is developed in this paper. This language is not designed for a specific simulator software. On the contrary, its purpose is to offer a general syntactic framework that could define a unified standard for membrane computing, covering a broad variety of models. At the present stage, P-Lingua can only handle $\mathrm{P}$ systems with active membranes, although the authors intend to extend it to other models in the near future. P-Lingua allows to write programs in a friendly way, as its syntax is very close to standard scientific notation, and parameterized expressions can be used as shorthand for sets of rules. There is a built-in compiler that parses these human-style programs and generates XML documents that can be given as input to simulation tools, different plugins can be designed to produce specific adequate outputs for existing simulators. Furthermore, we present in this paper an integrated development environment that plays the role of interface where P-lingua programs can be written and compiled. We also present a simulator for the class of recognizer P systems with active membranes, and we illustrate it by following the writing, compiling and simulating processes with a family of $\mathrm{P}$ systems solving the SAT problem.
\end{abstract}

\section{Introduction}

Membrane computing (or cellular computing) is an emerging branch of Natural Computing that was introduced by Gh. Păun [5]. The main idea is to consider biochemical processes taking place inside living cells from a computational point of view, in a way that provides a new nondeterministic model of computation.

The initial definition of this computing paradigm is very flexible, and many different models have been defined and investigated in the area: $\mathrm{P}$ systems with symport/antiport rules, with active membranes, with catalysts, with promoters/inhibitors, etc. There were some attempts to establish a common formalization covering most of the existing models (see e.g. [2]), but the membrane computing community is still using specific syntax and semantics depending on the model they work with. 
This diversification also exists in what concerns the development of software applications for the simulation of $\mathrm{P}$ systems (see [3], [11]), as such applications are usually focused on, and adapted for, particular cases, making it difficult to work on generalizations.

It is convenient to unify standards (specifications that regulate the performance of specific processes in order to guarantee their interoperability) and to implement the necessary tools and libraries in order to give the first steps towards a next generation of applications.

When designing software for membrane computing, one has to precisely describe the variant specification that is to be simulated. This task is hard if we need to handle families of $\mathrm{P}$ systems where the set of rules, the alphabet, the initial contents and even the membrane structure depend on the value assigned to some initial parameters. In existing software, several options have been implemented: plain text files with a determined format, XML documents, graphical user interfaces, etc. As mentioned above, most of these solutions are adapted to specific models or to the specific purpose of the software.

In this paper we propose a programming language, called P-Lingua, whose programs define $\mathrm{P}$ systems in a parametric and modular way. After assigning values to the initial parameters, the compilation tool generates an XML document associated with the corresponding $\mathrm{P}$ system from the family, and furthermore it checks possible programming errors (both lexical/syntactical and semantical). Such documents can be integrated into other applications, thus guaranteeing interoperability. More precisely, in the simulators framework, the XML specification of a $\mathrm{P}$ system can be translated into an executable representation.

We present a practical application of P-Lingua giving a simulator for recognizer $\mathrm{P}$ systems with active membranes that receives as input an XML document generated by the compiler and that allows us to simulate a computation, obtaining the correct answer of the system (due to the confluence of it), and a text file with a detailed step-by-step report of the computation. We also show an integrated development environment that plays the role of interface where P-Lingua programs can be written and compiled.

The paper is structured as follows. In Section 2 several definitions and concepts are given for the sake of selfcontainment of the paper. The next section introduces the P-Lingua programming language, and the syntax for $\mathrm{P}$ systems with active membranes is specified. A solution to the SAT problem using PLingua is implemented in Section 4. The compilation tool for the language is presented in the next section. In Section 6 we present an integrated development environment for P-Lingua. Section 7 presents a simulator for recognizer $\mathrm{P}$ systems with active membranes. Finally, some conclusions and ideas for future work are presented.

\section{Preliminaries}

Polynomial time solutions to computationally hard problems in membrane computing are achieved by trading time for space. This is inspired by the capability 
of cells to produce an exponential number of new membranes in linear time. There are many ways a living cell can produce new membranes: mitosis (cell division), autopoiesis (membrane creation), gemmation, etc. Following these inspirations a number of different variants of $\mathrm{P}$ systems has arisen, and many of them proved to be computationally universal.

For the sake of simplicity, we shall focus in this paper on a model, $P$ systems with active membranes. It is a construct of the form $\Pi=$ $\left(O, H, \mu, \omega_{1}, \ldots, \omega_{m}, R\right)$, where $m \geq 1$ is the initial degree of the system; $O$ is the alphabet of objects, $H$ is a finite set of labels for membranes; $\mu$ is a membrane structure, consisting of $m$ membranes injectively labelled with elements of $H, \omega_{1}, \ldots, \omega_{m}$ are strings over $O$, describing the multisets of objects placed in the $m$ regions of $\mu$; and $R$ is a finite set of rules, where each rule is of one of the following forms:

(a) $[a \rightarrow v]_{h}^{\alpha}$ where $h \in H, \alpha \in\{+,-, 0\}$ (electrical charges), $a \in O$ and $v$ is a string over $O$ describing a multiset of objects associated with membranes and depending on the label and the charge of the membranes (object evolution rules);

(b) $a[]_{h}^{\alpha} \rightarrow[b]_{h}^{\beta}$ where $h \in H, \alpha, \beta \in\{+,-, 0\}, a, b \in O$ (send-in communication rules). An object is introduced in the membrane, possibly modified, and the initial charge $\alpha$ is changed to $\beta$;

(c) $[a]_{h}^{\alpha} \rightarrow[]_{h}^{\beta} b$ where $h \in H, \alpha, \beta \in\{+,-, 0\}, a, b \in O$ (send-out communication rules). An object is sent out of the membrane, possibly modified, and the initial charge $\alpha$ is changed to $\beta$;

(d) $[a]_{h}^{\alpha} \rightarrow b$ where $h \in H, \alpha \in\{+,-, 0\}, a, b \in O$ (dissolution rules). A membrane with a specific charge is dissolved in reaction with a (possibly modified) object;

(e) $[a]_{h}^{\alpha} \rightarrow[b]_{h}^{\beta}[c]_{h}^{\gamma}$ where $h \in H, \alpha, \beta, \gamma \in\{+,-, 0\}, a, b, c \in O$ (division rules). A membrane is divided into two membranes. The objects inside the membrane are replicated, except for $a$, that may be modified in each membrane.

Rules are applied according to the following principles:

- All the elements which are not involved in any of the operations to be applied remain unchanged.

- Rules associated with label $h$ are used for all membranes with this label, no matter whether the membrane is an initial one or whether it was generated by division during the computation.

- Rules from (a) to (e) are used as usual in the framework of membrane computing, i.e. in a maximal parallel way. In one step, each object in a membrane can only be used by at most one rule (non-deterministically chosen), but any object which can evolve by a rule must do it (with the restrictions indicated below).

- Rules (b) to (e) cannot be applied simultaneously in a membrane in one computation step. 
- An object $a$ in a membrane labelled with $h$ and with charge $\alpha$ can trigger a division, yielding two membranes with label $h$, one of them having charge $\beta$ and the other one having charge $\gamma$. Note that all the contents present before the division, except for object $a$, can be the subject of rules in parallel with the division. In this case we consider that in a single step two processes take place: "first" the contents are affected by the rules applied to them, and "after that" the results are replicated into the two new membranes.

- If a membrane is dissolved, its content (multiset and interior membranes) becomes part of the immediately external one. The skin is never dissolved.

The so-called recognizer $\mathrm{P}$ systems were introduced in [6], and constitute the natural framework to study the solvability of decision problems, since deciding whether an instance has an affirmative or negative answer is equivalent to deciding if a string belongs or not to the language associated with the problem.

In the literature, recognizer $\mathrm{P}$ systems are associated in a natural way with $\mathrm{P}$ systems with input. The data related to an instance of the decision problem has to be provided to the $\mathrm{P}$ system in order for it to compute the appropriate answer. This is done by codifying each instance as a multiset placed in an input membrane. The output of the computation, yes or no, is sent to the environment.

A $P$ system with input is a tuple $\left(\Pi, \Sigma, i_{\Pi}\right)$, where: (a) $\Pi$ is a $\mathrm{P}$ system, with working alphabet $\Gamma$, with $p$ membranes labelled by $1, \ldots, p$, and initial multisets $\omega_{1}, \ldots, \omega_{p}$ associated with them; (b) $\Sigma$ is an (input) alphabet strictly contained in $\Gamma$; the initial multisets are over $\Gamma \backslash \Sigma$; and (c) $i_{\Pi}$ is the label of a distinguished (input) membrane.

For each multiset, $m$, over $\Sigma$, the initial configuration of $\left(\Pi, \Sigma, i_{\Pi}\right)$ with input $m$ is $\left(\mu, \omega_{1}, \ldots, \omega_{i_{\Pi}}+m, \ldots, \omega_{p}\right)$.

A recognizer $P$ system is a $\mathrm{P}$ system with input, $\left(\Pi, \Sigma, i_{\Pi}\right)$, and with external output such that:

(a) The working alphabet contains two distinguished elements, yes and no.

(b) All computations halts.

(c) If $\mathcal{C}$ is a computation of $\Pi$, then either the object yes or the object no (but no both) must have been released into the environment, and only in the last step of the computation.

We say that $\mathcal{C}$ is an accepting computation (respectively, rejecting computation) if the object yes (respectively, no) appears in the external environment associated with the corresponding halting configuration of $C$.

\section{The P-Lingua programming language}

A programming language is an artificial language that can be used to control the behavior of a machine, particularly a computer, but it can be used also to define a model of a machine that can be translated into an executable representation by a simulation tool.

Programming languages are defined by syntactic and semantic rules which describe their structure and their meaning, respectively. 
The P-Lingua programming language intends to define a broad variety of $\mathrm{P}$ system models. At the present stage, P-Lingua can only define P systems with active membranes, but other models will be added to the language specification in future works.

What follows is the syntax of the language for $\mathrm{P}$ systems with active membranes (originally presented at [1]).

\subsection{Valid identifiers}

We say that a sequence of characters forms a valid identifier if it does not begin with a numeric character and it is composed by characters from the following:

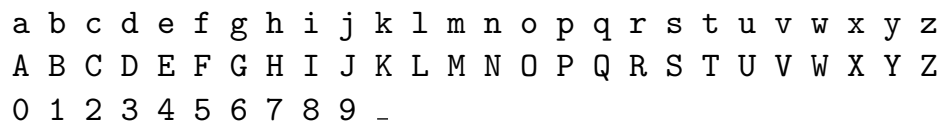

Valid identifiers are widely used in the language: to define module names, parameters, indexes, membrane labels and alphabet objects.

The following text strings are reserved words in the language: def, call, @mu, @ms, main, -->, \# and they cannot be used as valid identifiers.

\subsection{Identifiers for electrical charges}

In P-Lingua, we can consider electrical charges by using the + and - symbols for positive and negative charges, respectively, and no one for neutral charge. It is worth mentioning that polarizationless $\mathrm{P}$ systems are included.

\subsection{Variables}

Two kind of variables are permitted in P-Lingua:

- indexes

- Parameters

Variables are used to store numeric values and their names are valid identifiers. We use 32 bits (signed), this allows a range from $-2^{31}$ to $2^{31}-1$.

\subsection{Numeric expressions}

Numeric expressions can be written by using the $*$ (multiplication), / (division), $\%$ (modulo), + (addition), - (subtraction) operators with integer numbers or variables, along with the use of parentheses. 


\subsection{Objects}

The objects of the alphabet of a $\mathrm{P}$ system are written using valid identifiers, and the inclusion of sub-indexes is permitted. For example, $x_{i, 2 n+1}$ and $Y e s$ are written as $x\{i, 2 * n+1\}$ and Yes, respectively.

The multiplicity of an object is represented by using the $*$ operator. For example, $x_{i}^{2 n+1}$ is written as $\mathrm{x}\{\mathrm{i}\} *(2 * \mathrm{n}+1)$.

\subsection{Modules definition}

Similarities between various solutions to NP-complete numerical problems by using families of recognizer $\mathrm{P}$ systems are discussed in [4]. Also, a cellular programming language is proposed based on libraries of subroutines. Using these ideas, a P-Lingua program consists of a set of programming modules that can be used more times by the same, or other, programs.

The syntax to define a module is the following.

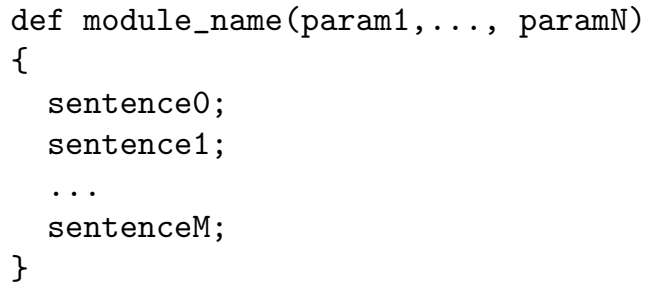

The name of a module, module_name, must be a valid and unique identifier. The parameters must be valid identifiers and cannot appear repeated. It is possible to define a module without parameters. Parameters have a numerical value that is assigned at the module call (see below).

All programs written in P-Lingua must contain a main module without parameters. The compiler will look for it when generating the XML file.

In $\mathrm{P}$-Lingua there are sentences to define the membranes configuration of a $\mathrm{P}$ system, to specify multisets, to define rules and to make calls to other modules. Next, let us see how such sentences are written.

\subsection{Module calls}

In P-Lingua, modules are executed by using calls. The format of a sentence that calls a module for some specific values of its parameters is given next:

call module name (value1, ..., valueN);

where value $i$ is an integer number or a variable. 


\subsection{Definition of the initial membrane structure of a $P$ system}

In order to define the initial membrane structure of a $\mathrm{P}$ system, the following sentence must be written:

$@ \mathrm{mu}=\operatorname{expr}$

where expr is a sequence of matching square brackets representing the membrane structure, including some identifiers that specify the label and the electrical charge of each membrane.

Examples:

1. $\left.\left[[]_{2}^{0}\right]_{1}^{0} \equiv @ \mathrm{mu}=[]^{\prime}, 2\right] ' 1$

2. $\left[[]_{b}^{0}[]_{c}^{-}\right]_{a}^{+} \equiv \mathrm{Qmu}=+\left[[]^{\prime} \mathrm{b},-[]^{\prime} \mathrm{c}\right]{ }^{\prime} \mathrm{a}$

\subsection{Definition of multisets}

The next sentence defines the initial multiset associated to the membrane labelled by label.

$$
\text { @ms }(\text { label })=\text { list_of_objects; }
$$

where label is a valid identifier or a natural number that represents a label of the structure of membranes and list_of_objects is a comma-separated list of objects. The character \# is used to represent the empty multiset.

\subsection{Union of multisets}

P-Lingua allows to define the union of two multisets (recall that the input multiset is added to the initial multiset of the input membrane) by using a sentence with the following format.

@ms (label) += list_of_objects;

\subsection{Definition of rules}

1. The format to define evolution rules of type $[a \rightarrow v]_{h}^{\alpha}$ is given next:

$\alpha[\mathrm{a}-->\mathrm{v}]$ 'h

2. The format to define send-in communication rules of type $a[]_{h}^{\alpha} \rightarrow[b]_{h}^{\beta}$ is given next:

$\mathrm{a} \alpha[]^{\prime} \mathrm{h}-->\beta[\mathrm{b}]$

3. The format to define send-out communication rules of type $[a]_{h}^{\alpha} \rightarrow b[]_{h}^{\beta}$ is given next:

$\alpha[\mathrm{a}]$ 'h $\rightarrow \beta[] \mathrm{b}$ 
4. The format to define division rules of type $[a]_{h}^{\alpha} \rightarrow[b]_{h}^{\beta}[c]_{h}^{\gamma}$ is given next:

$$
\alpha[\mathrm{a}] \text { ' } \mathrm{h}-->\beta[\mathrm{b}] \gamma[\mathrm{c}]
$$

5. The format to define dissolution rules of type $[a]_{h}^{\alpha} \rightarrow b$ is given next:

$$
\alpha[\mathrm{a}] \text { 'h } \rightarrow \mathrm{b}
$$

where:

$-\alpha, \beta$ and $\gamma$ are identifiers for electrical charges;

$-\mathrm{a}, \mathrm{b}$ and $\mathrm{c}$ are objects of the alphabet;

- $\mathrm{v}$ is a comma-separated list of objects that represents a multiset;

$-\mathrm{h}$ is a label (the symbol' always precedes a label name).

Some examples:

$-\left[x_{i, 1} \rightarrow r_{i, 1}^{4}\right]_{2}^{+} \equiv+[\mathrm{x}\{\mathrm{i}, 1\} \rightarrow \mathrm{r}\{\mathrm{i}, 1\} * 4]^{\prime} 2$

$-d_{k}[]_{2}^{0} \rightarrow\left[d_{k+1}\right]_{2}^{0} \equiv \mathrm{d}\{\mathrm{k}\}[]^{\prime} 2-->[\mathrm{d}\{\mathrm{k}+1\}]$

$-\left[d_{k}\right]_{2}^{+} \rightarrow[]_{2}^{0} d_{k} \equiv+[\mathrm{d}\{\mathrm{k}\}]^{\prime} 2-->[] \mathrm{d}\{\mathrm{k}\}$

$-\left[d_{k}\right]_{2}^{0} \rightarrow\left[d_{k}\right]_{2}^{+}\left[d_{k}\right]_{2}^{-} \equiv[\mathrm{d}\{\mathrm{k}\}]^{\prime} 2-->+[\mathrm{d}\{\mathrm{k}\}]-[\mathrm{d}\{\mathrm{k}\}]$

$-[a]_{2}^{-} \rightarrow b \equiv-[\mathrm{a}] ' 2-->\mathrm{b}$

\subsection{Parametric sentences}

In P-Lingua, it is possible to define parametric sentences by using the next format:

sentence : range $1, \ldots$, rangeN;

where sentence is a sentence of the language, or a sequence of sentences in brackets, and range $1, \ldots$, rangeN is a comma-separated list of ranges with the format:

min_value $<=$ index $<=$ max_value

where min_value and max_value are numeric expressions, integer numbers or variables, and index is a variable that can be used in the context of the sentence. It is possible to use the operator $<$ instead of $<=$.

The sentence will be repeated for each possible values of each index.

Some examples of parametric sentences:

1. $\left[d_{k}\right]_{2}^{0} \rightarrow\left[d_{k}\right]_{2}^{+}\left[d_{k}\right]_{2}^{-}: 1 \leq k \leq n \equiv$ $[\mathrm{d}\{\mathrm{k}\}]]^{\prime} 2->+[\mathrm{d}\{\mathrm{k}\}]-[\mathrm{d}\{\mathrm{k}\}]: 1<=\mathrm{k}<=\mathrm{n}$;

2. $\left[x_{i, j} \rightarrow x_{i, j-1}\right]_{2}^{+}: 1 \leq i \leq m, 2 \leq j \leq n \equiv$ $+[x\{i, j\} \rightarrow x\{i, j-1\}]^{\prime} 2: 1<=i<=m, 2<=j<=n$; 


\subsection{Inclusion of comments}

The programs in P-Lingua can be commented by writing phrases into the text strings $/ *$ and $* /$.

\section{Implementation of a solution to SAT}

In this section, we present a solution to the SAT problem using recognizer $\mathrm{P}$ systems with active membranes, given by M.J. Pérez-Jiménez et al. [7].

For each $(m, n) \in \mathbb{N}^{2}$, we consider the P system $(\Pi(\langle m, n\rangle), \Sigma(m, n), i(m, n))$, where

$-\Sigma(m, n)=\left\{x_{i, j}, \bar{x}_{i, j}: 1 \leq i \leq m, 1 \leq j \leq n\right\}$

$-i(m, n)=2$

- $\Pi(\langle m, n\rangle)=\left(\Gamma(m, n),\{1,2\},\left[[]_{2}\right]_{1}, w_{1}, w_{2}, R\right)$, is defined as follows:

- $\Gamma(m, n)=\Sigma(m, n) \cup\left\{c_{k}: 1 \leq k \leq m+2\right\} \cup$

$\left\{d_{k}: 1 \leq k \leq 3 n+2 m+3\right\} \cup$

$\left\{r_{i, k}: 0 \leq i \leq m, 1 \leq k \leq m+2\right\} \cup\{e, t\} \cup\{Y e s, N o\}$

- $w_{1}=\emptyset$

- $w_{2}=\left\{d_{1}\right\}$

- The set of rules, $R$, is given by:

$$
\begin{aligned}
& \left\{\left[d_{k}\right]_{2}^{0} \rightarrow\left[d_{k}\right]_{2}^{+}\left[d_{k}\right]_{2}^{-}: 1 \leq k \leq n\right\} \\
& \left\{\left[x_{i, 1} \rightarrow r_{i, 1}\right]_{2}^{+},\left[\bar{x}_{i, 1} \rightarrow r_{i, 1}\right]_{2}^{-}: 1 \leq i \leq m\right\} \\
& \left\{\left[x_{i, 1} \rightarrow \lambda\right]_{2}^{-},\left[\bar{x}_{i, 1} \rightarrow \lambda\right]_{2}^{+}: 1 \leq i \leq m\right\} \\
& \left\{\left[x_{i, j} \rightarrow x_{i, j-1}\right]_{2}^{+},\left[x_{i, j} \rightarrow x_{i, j-1}\right]_{2}^{-}: 1 \leq i \leq m, 2 \leq j \leq n\right\} \\
& \left\{\left[\bar{x}_{i, j} \rightarrow \bar{x}_{i, j-1}\right]_{2}^{+},\left[\bar{x}_{i, j} \rightarrow \bar{x}_{i, j-1}\right]_{2}^{-}: 1 \leq i \leq m, 2 \leq j \leq n\right\} \\
& \left\{\left[d_{k}\right]_{2}^{+} \rightarrow[]_{2}^{0} d_{k},\left[d_{k}\right]_{2}^{-} \rightarrow[]_{2}^{0} d_{k}: 1 \leq k \leq n\right\} \\
& \left\{d_{k}[]_{2}^{0} \rightarrow\left[d_{k+1}\right]_{2}^{0}: 1 \leq k \leq n-1\right\} \\
& \left\{\left[r_{i, k} \rightarrow r_{i, k+1}\right]_{2}^{0}: 1 \leq i \leq m, 1 \leq k \leq 2 n-1\right\} \\
& \left\{\left[d_{k} \rightarrow d_{k+1}\right]_{1}^{0}: n \leq k \leq 3 n-3\right\} ;\left[d_{3 n-2} \rightarrow d_{3 n-1} e\right]_{1}^{0} \\
& e[]_{2}^{0} \rightarrow\left[c_{1}\right]_{2}^{+} ;\left[d_{3 n-1} \rightarrow d_{3 n}\right]_{1}^{0} \\
& \left\{\left[d_{k} \rightarrow d_{k+1}\right]_{1}^{0}: 3 n \leq k \leq 3 n+2 m+2\right\} \\
& {\left[r_{1,2 n}\right]_{2}^{+} \rightarrow[]_{2}^{-} r_{1,2 n} ; \quad\left\{\left[r_{i, 2 n} \rightarrow r_{i-1,2 n}\right]_{2}^{-}: 1 \leq i \leq m\right\}} \\
& r_{1,2 n}[]_{2}^{-} \rightarrow\left[r_{0,2 n}\right]_{2}^{+} \\
& \left\{\left[c_{k} \rightarrow c_{k+1}\right]_{2}^{-}: 1 \leq k \leq m\right\} \\
& {\left[c_{m+1}\right]_{2}^{+} \rightarrow[]_{2}^{+} c_{m+1} ; \quad\left[c_{m+1} \rightarrow c_{m+2} t\right]_{1}^{0}} \\
& {[t]_{1}^{0} \rightarrow[]_{1}^{+} t ; \quad\left[c_{m+2}\right]_{1}^{+} \rightarrow[]_{1}^{-} Y e s ; \quad\left[d_{3 n+2 m+3}\right]_{1}^{0} \rightarrow[]_{1}^{+} N o}
\end{aligned}
$$




\subsection{Implementation}

The following is the code of the program written in P-Lingua that specifies a family of $\mathrm{P}$ systems solving the SAT problem.

Objects of the form $\bar{x}_{i, j}$ are written as $\mathrm{nx}\{\mathrm{i}, \mathrm{j}\}$.

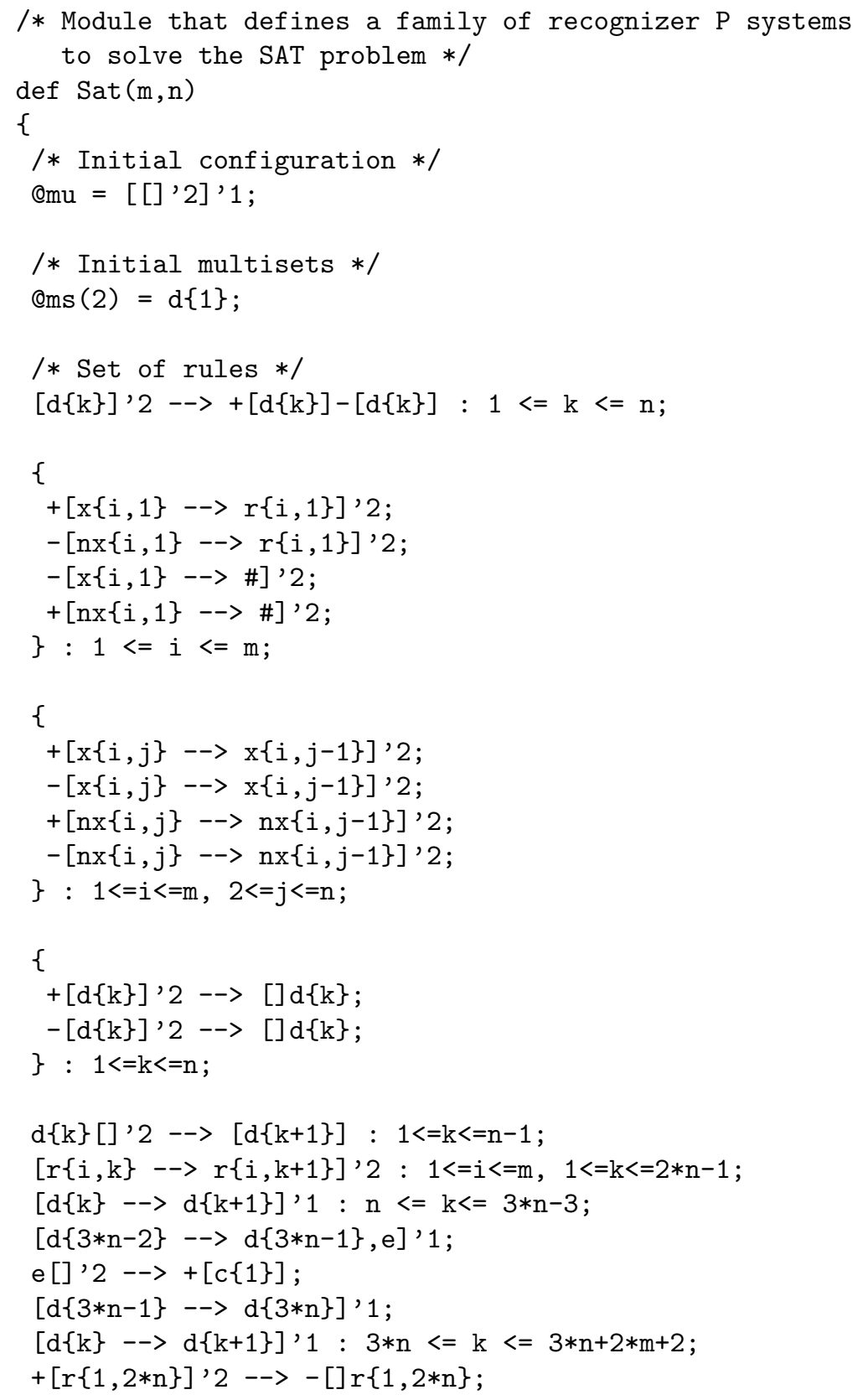




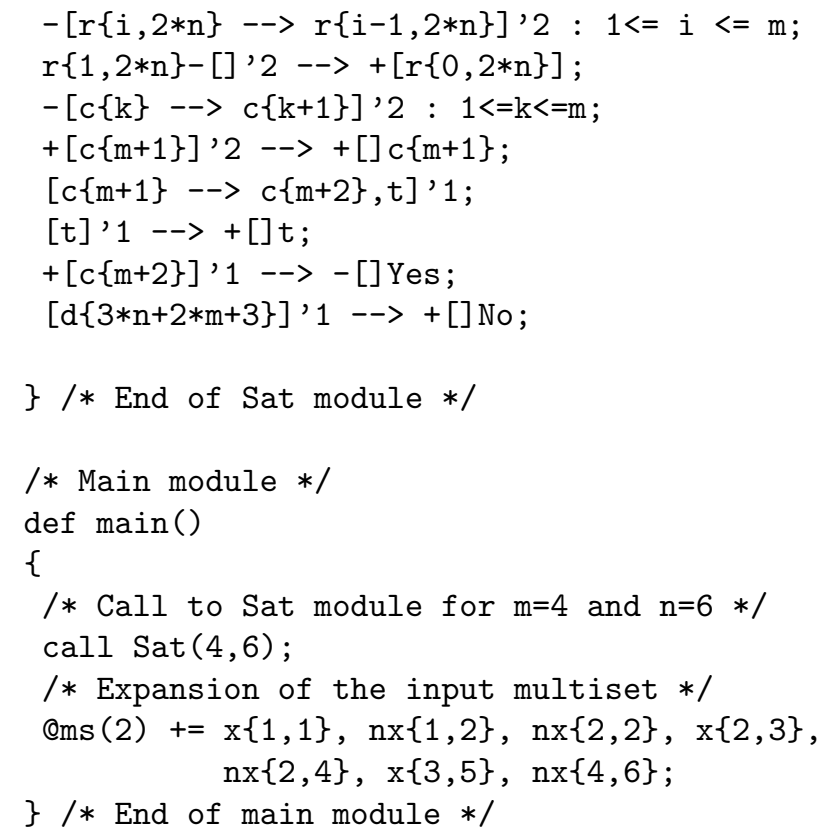

The module main is instantiated with the formula

$$
\varphi \equiv\left(x_{1}+\bar{x}_{2}\right)\left(\bar{x}_{2}+x_{3}+\bar{x}_{4}\right) x_{5} \bar{x}_{6}
$$

where $n=6, m=4$ and the input multiset: $x_{1,1}, \bar{x}_{1,2}, \bar{x}_{2,2}, x_{2,3}, \bar{x}_{2,4}, x_{3,5}, \bar{x}_{4,6}$.

\section{The compilation tool}

Programming languages are associated with compilation tools, which are computer programs that translate text written in a programming language into another language. The original text is usually called the source code whereas the output is called the object code. Commonly the output has a form suitable for being processed by other programs or for being executed by the computer, but it may as well be a human-readable text file.

We have developed a compilation tool that is able to translate programs written in P-lingua into XML documents, after having assigned values to some initial parameters. Moreover, plugins can be designed and added to produce object code with different formats.

Recall that a P-lingua program can encode a family of $\mathrm{P}$ systems (with the help of some parameters) in a flexible manner, whereas the object code generated by the compilation tool specifies only a single $\mathrm{P}$ system of the family. In this way, the applications that accept that object code do not need to process parametric systems, and hence their implementation is much easier. 
The eXtensible Markup Language (XML) is a general-purpose specification for creating custom markup languages. It is classified as an extensible metalanguage because it allows the users to define their own elements. Its primary purpose is to facilitate the sharing of structured data across different information systems. It is worth mentioning that the SBML (Systems Biology Markup Language) is a XML language encoding the main components of biochemical networks. It is used by several existing simulators for $\mathrm{P}$ systems (see the software link at [11]).

The complete syntax of the XML language generated by the compilation tool for $\mathrm{P}$ systems with active membranes can be found at [1].

The tool may be executed from the command line as follows:

plingua input_file -xml output_file [-v verbosity_level] [-h]

The text file input_file contains the program (written in P-lingua) that we want to be compiled, and output_file is the name of the XML file that is generated. Optional arguments are in brackets: the option -v verbosity_level is a number between 0 and 5 indicating the level of detail of the messages shown during the compilation process, and the option - $\mathrm{h}$ displays some help information.

\section{An integrated development environment}

An integrated development environment (IDE) is a software application that provides comprehensive facilities to computer programmers for software development. Usually, an IDE consists of a source code editor, a compiler and/or interpreter, a debugger, and other useful tools.

Typically an IDE is devoted to a specific programming language, so as to provide a feature set which most closely matches the programming paradigms of the language. In this sense, we have developed an IDE for P-Lingua by using the Java language. This application provides an environment to write and debug programs in $\mathrm{P}$-Lingua for $\mathrm{P}$ systems with active membranes, and it can be updated by adding plugins to accept future versions of the language. The IDE can also be used as a simulation tool for P-Lingua programs.

This application includes a source code editor with syntax highlighting which is a feature that displays text source code in different colors and fonts, as both structures and syntax errors are visually distinct. With this editor, it is also possible to generate P-Lingua programs composed of several single files.

A compilation tool is included to check possible programming errors and to generate XML files that can be used in third-part applications.

A simulation tool for debugging is included in order to aid the researcher in the task of designing new $\mathrm{P}$ systems. This tool provides simulations by using an interactive step-by-step mode. The user can choose between simulation of one or several steps, or let the simulation run until a halting state. A lot of information is given in each step of the simulation: a tree-view of the membranes structure, complete information of the multisets and the set of rules selected to 
be executed. The user can also choose between different non-deterministic ways of computation, or let the software select one.

\section{A simulator for recognizer $P$ systems with active membranes}

The act of simulating generally entails representing certain key characteristics or behaviors of some physical, or abstract, system. We must distinguish a simulation tool from an emulation tool: this duplicates the functions of one system by using a different system, so that the second system behaves like (and appears to be) the emulated system. With the current technology, we cannot emulate the functionality of a cellular machine (a membrane system) by using a conventional computer to solve instances of NP-hard problems in a polynomial time, but we can simulate these cellular machines for research purposes, even if the simulation is not done in a polynomial time.

The P system computations are massively parallel. One of the most common programming methods to simulate real parallelism in a conventional computer with a single processor is to use multithreading. A thread in this sense is a thread of execution. Threads are a way for a program to fork (or split) itself into two or more simultaneously (or pseudo-simultaneously) running tasks. Multiple threads can be executed in parallel on a single computer. This multithreading generally occurs by time-division multiplexing where the processor switches between different threads. This context switching can happen so fast as to give the illusion of parallelism to an end-user. On a multiprocessor or multi-core system, threading can be achieved via multiprocessing, wherein different threads can literally run simultaneously on different processors or cores.

As a first practical application of the P-lingua programming language, we have implemented a simulator for recognizer $\mathrm{P}$ systems with active membranes that takes as input an XML document generated by the P-lingua compiler and runs one of the possible computations that the $\mathrm{P}$ system may follow, obtaining the answer that the system outputs to its environment, and a text file with a detailed step-by-step report of the computation.

The system requirements are the same as in the case of the P-lingua compiler. The simulator is launched from the command line as follows:

$$
\text { plingua_sim input_xml [-o output_file] }
$$

where input_xml is an XML document formatted as discussed in this paper, and output_file is the name of the file where the report about the simulated computation will be saved.

\subsection{Simulation of a solution to the SAT problem}

We now show an execution of the simulator running on the XML document obtained after compiling the P-lingua program described in Section 4.1. The 
results have been obtained on an AMD Sempron machine, at $2.8 \mathrm{Ghz}$ and with $512 \mathrm{Mb}$ of RAM memory.

The command used to execute the simulation is:

plingua_sim sat.xml -o info.txt

The simulation ends when no more rules can be applied, and then the following information is displayed:

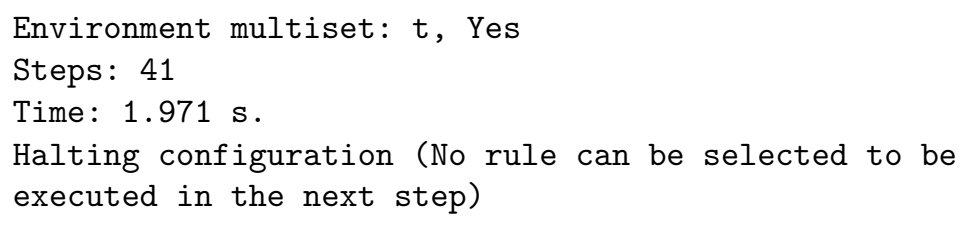

Thus, the computation of the $\mathrm{P}$ system spend 41 transition steps, and it took 1.971 seconds to simulate it until reaching a halting configuration (recall that we are simulating a parallel device on a sequential computer).

The file info.txt keeps detailed information about each configuration of the simulated computation. More precisely, the multisets and polarizations of all the membranes are listed, as well as the rules selected for execution at each transition step. The configurations are numbered (starting at 0), to keep track of the step of the computation that is being simulated. Some information about the CPU time is shown for each step, and the number of rules of each type that is executed. As an example, we give the information generated for the first two configurations.

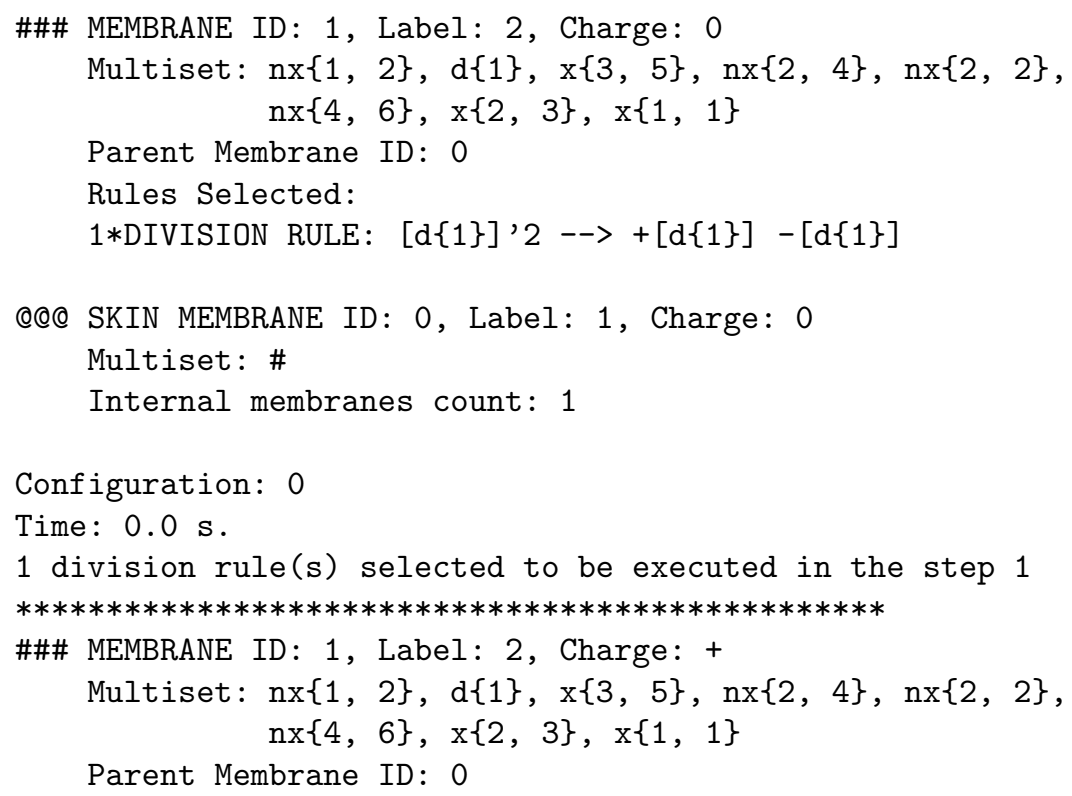




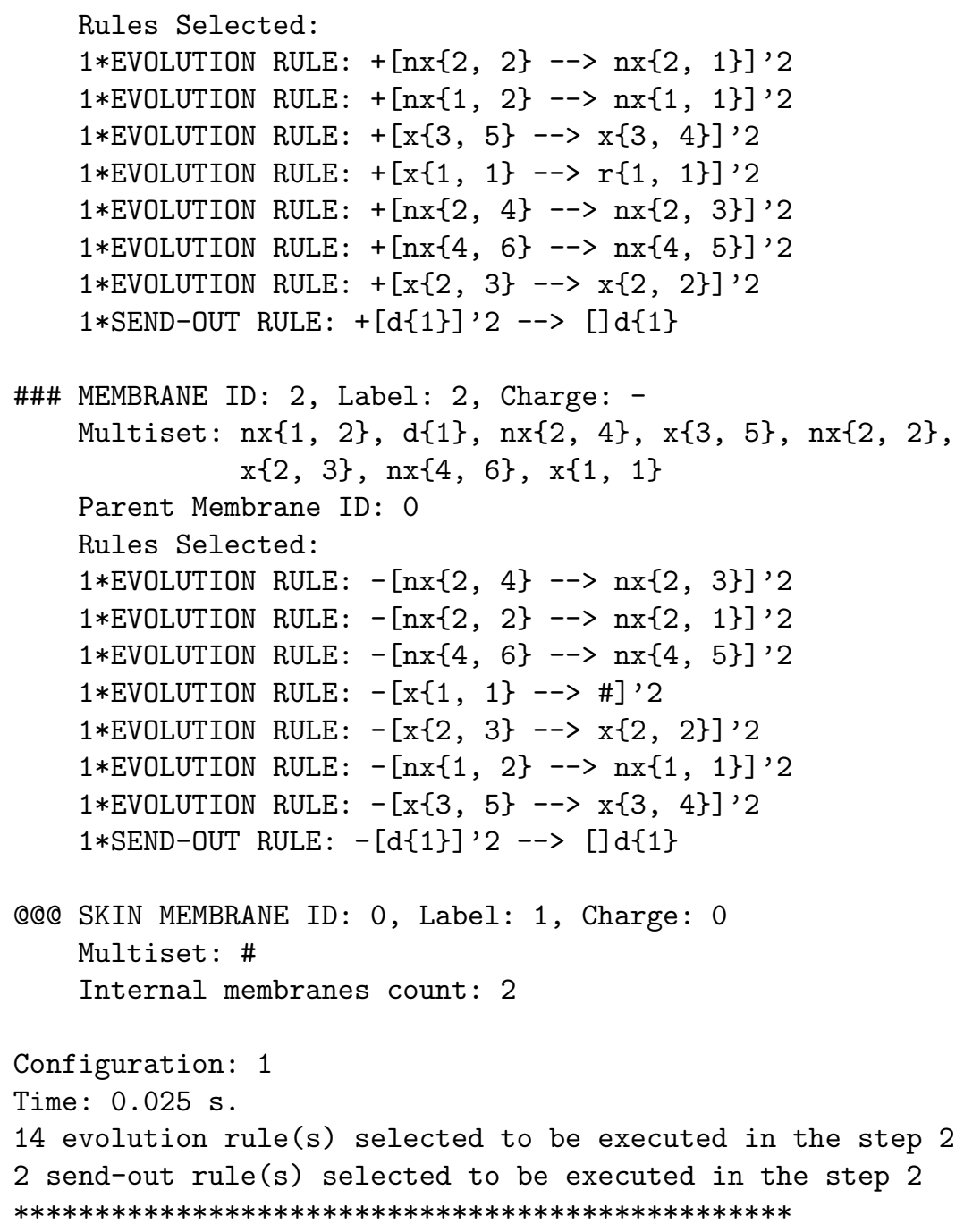

After simulating 41 transition steps, the halting configuration is described as follows:

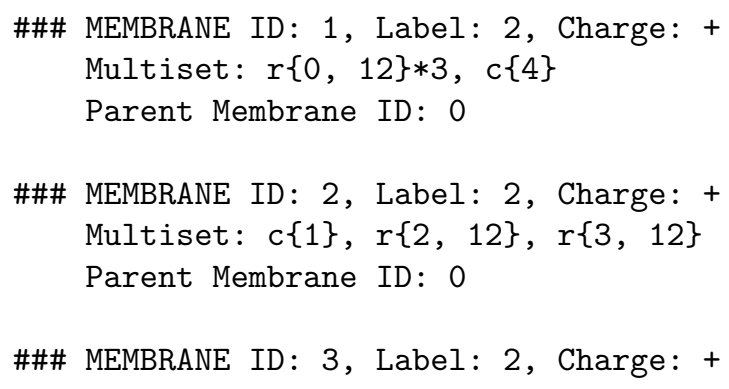




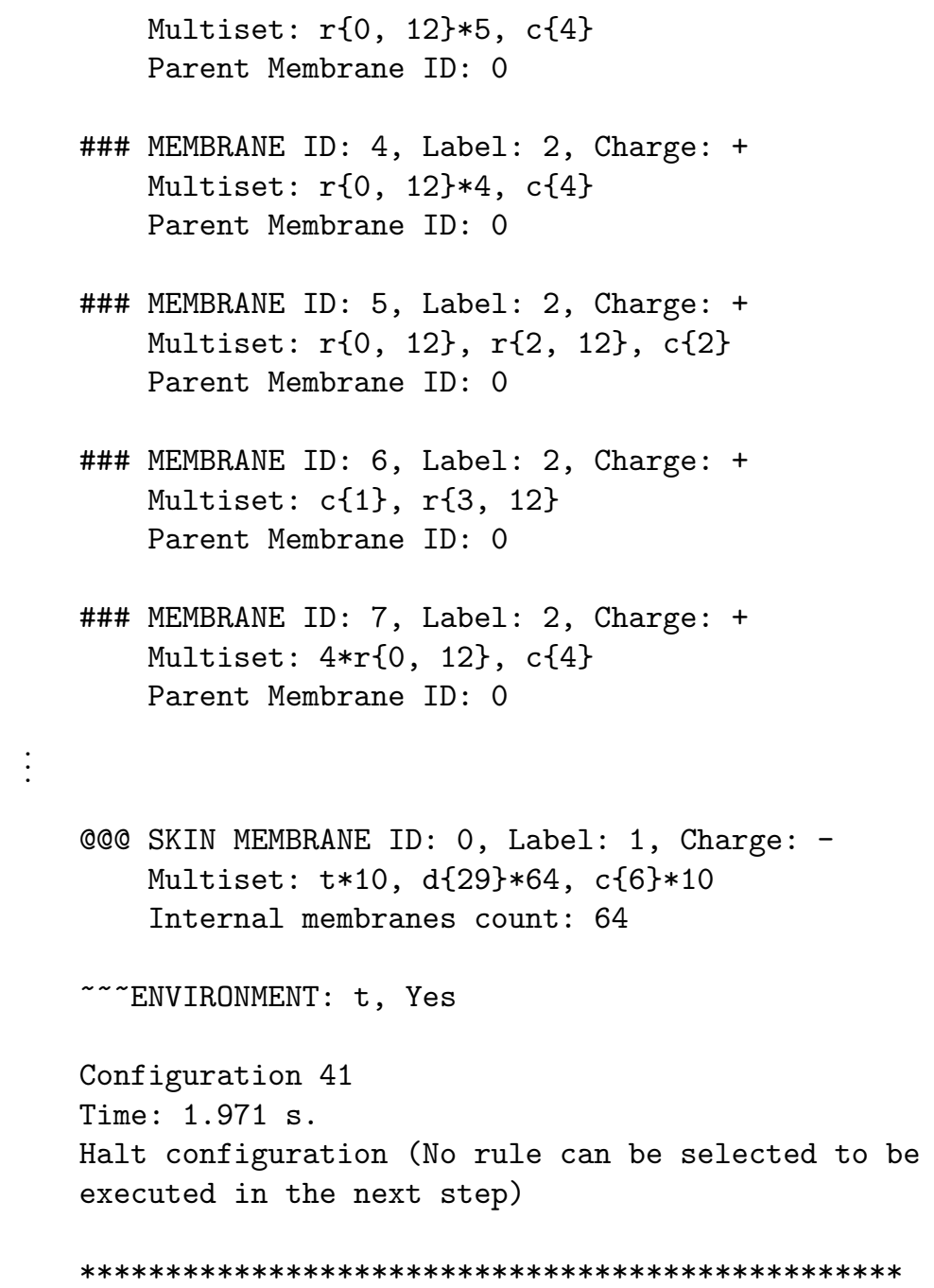

Note that there are 64 different membranes labelled by 2 in this configuration, although for the sake of simplicity we show only seven of them.

\section{Conclusions and future work}

In this paper we have presented a programming language for membrane computing, $P$-Lingua, together with a compiler that generates XML documents, an integrated development environment and a simulator for a class of $\mathrm{P}$ systems, namely recognizer $\mathrm{P}$ systems with active membranes.

Using a programming language to define cellular machines is a concept in the development of applications for membrane computing that leads to a standardization with the following advantages: 
- Users can define cellular machines in a modular and parametric way by using an easy-to-learn programming language.

- It is possible to define libraries of modules that can be shared among users to facilitate the design of new programs.

- This method to define P systems is decoupled from its applications and the same P-Lingua programs can be used in different software environments.

- By using compiling tools, the P-Lingua programs are translated to other file formats that can be interpreted by a large number of different applications.

The first version of $\mathrm{P}$-Lingua is presented for $\mathrm{P}$ systems with active membranes. In forthcoming versions, we will try to generalize the language so that other types of cellular devices can be also defined, for instance transition $\mathrm{P}$ systems and tissue $\mathrm{P}$ systems. In this sense, necessary plugins (software modules) for the IDE and the compilation tool must be developed also.

We have chosen an XML language as the output format because of the reasons exposed above. However, we are aware that for some applications it is not the most suitable format, due to the fact that XML does not include any method for compressing data, and therefore the text files can eventually become too large, which is a clear disadvantage for applications running on networks of processors. It would be convenient to improve the compiler (by adding plugins) so that it generates a larger variety of output formats, of special interest are compressed binary files or executable code (either in C or Java).

It is important to recall that the simulator presented here is designed to run in a conventional computer, having limited resources (RAM, CPU), and this leads to a bound on the size of the instances of computationally hard problems whose solutions can be successfully simulated. Moreover, conventional computers are not massively parallel devices, and therefore it seems that the inherent parallelism of $\mathrm{P}$ systems must be simulated by means of multithreading techniques. It would be interesting to design heuristics which help us to find good (short) computations.

These shortcomings lead us to the possibility of implementing a distributed simulator running on a network or cluster of processors, where the need of resources arising during the computation could be solved by adding further nodes to the network, thus moving towards massive parallelism.

The sofware presented in this paper and its source code can be freely downloaded from the software section on the website [12]. This software is under the GNU General Public License (GNU GPL) [8] and it is written in Java [9] using the lexical and syntactical analyzers provided by JavaCC [10]. The minimum system requirements are having a Java virtual machine (JVM) version 1.6.0 running in a Pentium III computer.

\section{Acknowledgment}

The authors acknowledge the support of the project TIN2006-13425 of the Ministerio de Educación y Ciencia of Spain, cofinanced by FEDER funds, as well as the support of the project of excellence TIC-581 of the Junta de Andalucía. 


\section{References}

1. D. Díaz-Pernil, I. Pérez-Hurtado, M.J. Pérez-Jiménez, A. Riscos-Núñez. PLingua: A programming Language for Membrane Computing. In Daniel DíazPernil, M.A. Gutiérrez-Naranjo, C. Graciani-Díaz, Gh. Păun, I. Pérez-Hurtado, A. Riscos-Núñez (eds) Proceedings of the 6th Brainstorming Week on Membrane Computing, Sevilla, Fénix Editora, (2008), 135-155.

2. R. Freund, S. Verlan. A Formal Framework for Static (Tissue) P Systems. Lecture Notes in Computer Science, 4860 (2007), 271-284.

3. M.A. Gutiérrez-Naranjo, M.J. Pérez-Jiménez, A. Riscos-Núñez. Available Membrane Computing Software. In G. Ciobau, Gh. Păun, M.J. Péréz-Jiménez (eds.) Applications of Membrane Computing, Natural Computing Series, Springer-Verlag, 2006. Chapter 15 (2006), pp. 411-436.

4. M.A. Gutiérrez-Naranjo, M.J. Pérez-Jiménez, A. Riscos-Núñez. Towards a programming language in cellular computing. Electronic Notes in Theoretical Computer Science, Elsevier B.V., 123 (2005), 93-110.

5. Gh. Păun. Computing with membranes. Journal of Computer and System Sciences, 61, 1 (2000), 108-143, and Turku Center for Computer Science-TUCS Report No 208.

6. M.J. Pérez-Jiménez, A. Romero-Jiménez, F. Sancho-Caparrini. Complexity classes in cellular computing with membranes. Natural Computing, 2, 3 (2003), 265-285.

7. M.J. Pérez-Jiménez, A. Romero-Jiménez, F. Sancho-Caparrini. A polynomial complexity class in $\mathrm{P}$ systems using membrane division. Journal of Automata, Languages and Combinatorics, 11, 4 (2006), 423-434.

8. The GNU General Public License: http://www.gnu.org/copyleft/gpl.html

9. Java web page: http://www.java.com/

10. JavaCC web page: https://javacc.dev.java.net/

11. P systems web page: http://ppage.psystems.eu/

12. Research Group on Natural Computing web page: http://www.gcn.us.es/ 